\title{
Evaluation of the Resistance of Spider Silk to Ultraviolet Irradiation
}

\author{
Shigeyoshi Osaki, ${ }^{\dagger}$ Keizo Yamamoto, Atsushi KaJiwara, ${ }^{*}$ and Mariko Murata** \\ Faculty of Medicine, Nara Medical University, Kashihara 634-8521, Japan \\ *Department of Materials Science, Nara University of Education, Nara 630-8528, Japan \\ **Faculty of Education, Shimane University, Matsue 690-8504, Japan
}

(Received March 2, 2004; Accepted May 8, 2004; Published August 15, 2004)

\begin{abstract}
The effects of ultraviolet (UV) irradiation on spider silk were studied in Nephila clavata draglines collected during different developmental stages in order to clarify the resistance of the draglines to sunlight. Electron spin resonance measurements revealed that photo-irradiation produced $\mathrm{C}_{\alpha}$-centered radicals of the protein molecules constituting the draglines. This was attributed to the photo-induced cleavage of chemical bonds. Greater numbers of radicals were produced in the draglines of mature spiders than in those of juveniles, and in the silks of silkworm compared with the draglines of spider. Spider silk is therefore more resistant to UV irradiation, which might be a consequence of the outside environment in which it functions. The spider silk is expected to be a useful next-generation material for textiles. [DOI 10.1295/polymj.36.623]

KEY WORDS Resistance / Spider Silk / Ultraviolet Rays / Radicals / ESR /
\end{abstract}

Previous studies have described many of the physicochemical (including mechanical, thermal and optical) properties of spider silk ${ }^{1-11}$ along with its safety. ${ }^{12-14}$ As a result spider silk is expected to be a useful next-generation material for textiles because of its outstanding physical properties. New genetic technology that allows the possibility of the mass production of spider silk has generated additional interest. ${ }^{15-17}$ It is important to understand the effects of exposure to ultraviolet (UV) radiation on spider silk, because resistance to UV irradiation influences the physical properties of textiles. However, despite these facts, the resistance of spider silk to UV irradiation has not yet been determined.

In Japan, juveniles of the golden web spider Nephila clavata hatch during late spring and reach sexual maturity, accompanied by a final ecdysis, in the summer months. Male spiders visit the orb webs of females in early autumn as part of the courtship process. After mating, the pregnant females lay their eggs inside silk cocoons during the late autumn and subsequently die. As shown in Figure 1, the weight (W) of female $N$. clavata spiders increases exponentially between the immature and reproductive stages, reaching a maximum in October.

Electron spin resonance (ESR) spectroscopy is a useful method for obtaining information on structures, concentrations, life times, and dynamics of organic radicals. ${ }^{18,19}$ If radical species are created by photo-induced cleavage during irradiation of light, the radicals would be detected by ESR spectroscopy and the spectra are expected to show characteristic season dependent property of the spider silk. Although one of the

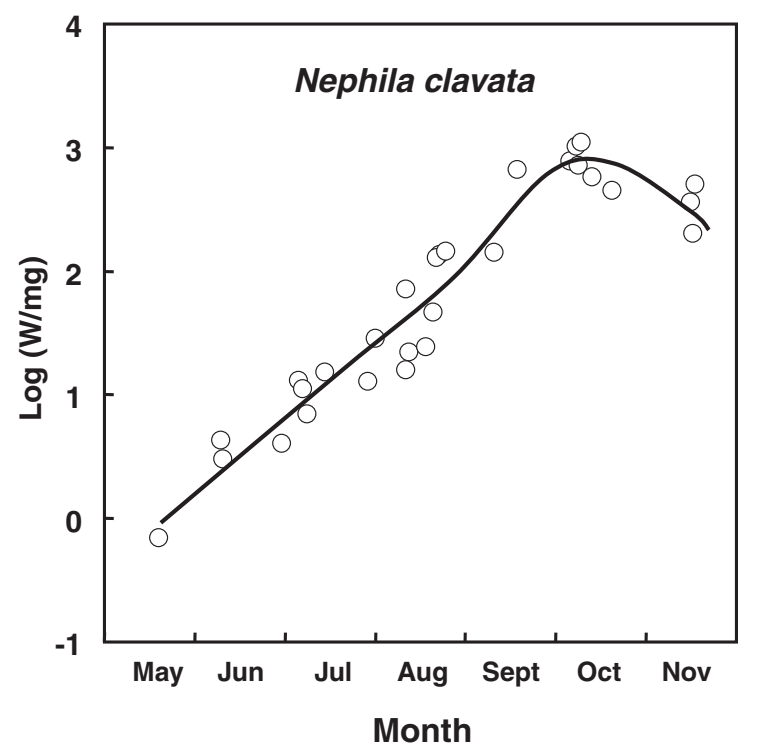

Figure 1. Log weight of $N$. clavata spiders at different stages of growth by month.

authors previously provided evidence of radical production in spider silk as a result of UV irradiation, ${ }^{20,21}$ it subsequently proved difficult to collect silk samples during different life-cycle stages to build on this initial research; however, we recently overcame this difficulty by developing a technique for collecting silk automatically from spiders.

The present paper therefore focused on the effects of UV irradiation on spider silk collected during different developmental stages. We investigated the resistance of spider silk to UV irradiation by using ESR method and compared the results with those ob-

${ }^{\dagger}$ To whom correspondence should be addressed (E-mail: s-osaki@naramed-u.ac.jp). 
tained using silk produced by silkworms (Bombyx mori).

\section{EXPERIMENTAL}

\section{Materials}

Spiders secrete draglines that function as lifelines when they hang in the air or tall from their webs. Here we prepared samples by winding the draglines, which was secreted from $N$. clavata spiders collected in Matsue, Japan, around a rectangular frame with a velocity of $\sim 50 \mathrm{~mm} / \mathrm{s}$ in the laboratory. Silks from silkworms were also used as samples.

\section{ESR Measurements and Optical Reflectance}

ESR spectra were measured using a JES RE-2X ESR spectrometer (JEOL, Ltd., Japan). Each spider silk sample was wound into a ball with a diameter of $c a .3 \mathrm{~mm}$ in order to minimize the effects of anisotropy. The small ball of wadded silk was then placed into a quartz cell with a diameter of $5 \mathrm{~mm}$. UV light was irradiated from an USHIO USD 500D ultrahigh-pressure mercury lamp of $500 \mathrm{~W}$ with a maximum wavelength of 250-365 nm (Ushio Inc., Japan). The quartz cell was $15 \mathrm{~cm}$ away from the mercury lamp. Radical concentrations were estimated compared with those of tetramethyl piperidinol powder containing $1 \%$ tetramethyl piperidinyloxyl. The $g$-value was determined to be 2.0057 using a $\mathrm{Mn}^{2+}$ marker.

Optical reflectance spectra were measured using a UV-3100 spectrometer (Shimadzu Corp., Japan). Dragline samples placed on a $\mathrm{BaSO}_{4}$ white standard board were irradiated vertically with wavelengths from 190 to $700 \mathrm{~nm}$, and the optical reflectance was measured at set time intervals.

\section{RESULTS}

Figure 2 shows the ESR spectra of dragline samples, which were collected from female $N$. clavata in Matsue on 25 September in 1996, measured before UV irradiation (origial) and after 5, 20 and $60 \mathrm{~min}$ of irradiation. The $g$-value of the radicals was determined as 2.0057 on the basis of the observed ESR spectra. This $g$-value is characteristic of $\mathrm{C}_{\alpha}$-centred radicals that are created on the peptide backbone of protein molecules. ${ }^{22}$ The results therefore suggest that UV irradiation induces the breaking of chemical bonds in the protein molecules that constitute spider silk.

The ESR spectra of $N$. clavata draglines changed with the duration of UV irradiation, and signal intensity increased over time. The signal intensity of an ESR spectrum reflects the quantity of radicals present; therefore, the increased signal intensity corresponded to the degradation of the draglines.

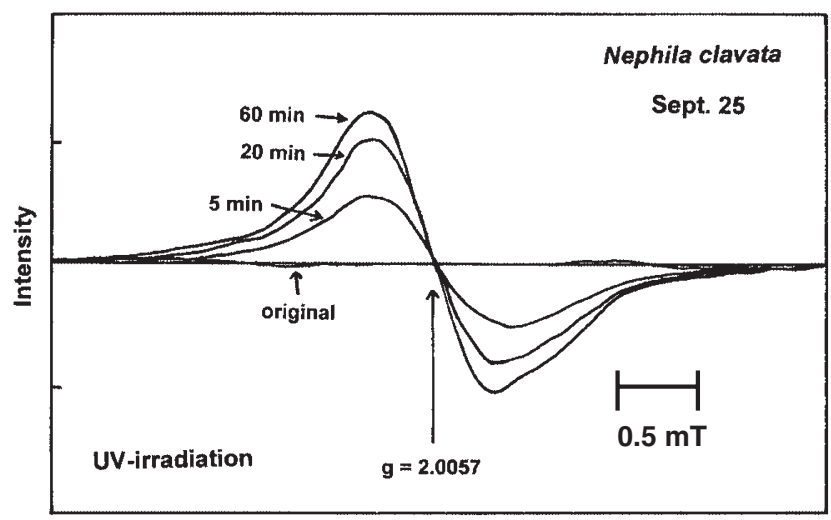

Figure 2. ESR spectra of the draglines of female $N$. clavata collected in Matsue, Japan on 25 September, measured before UV irradiation (0) and after 5, 20 and $60 \mathrm{~min}$ of UV irradiation. The $g$-value was 2.0057 .

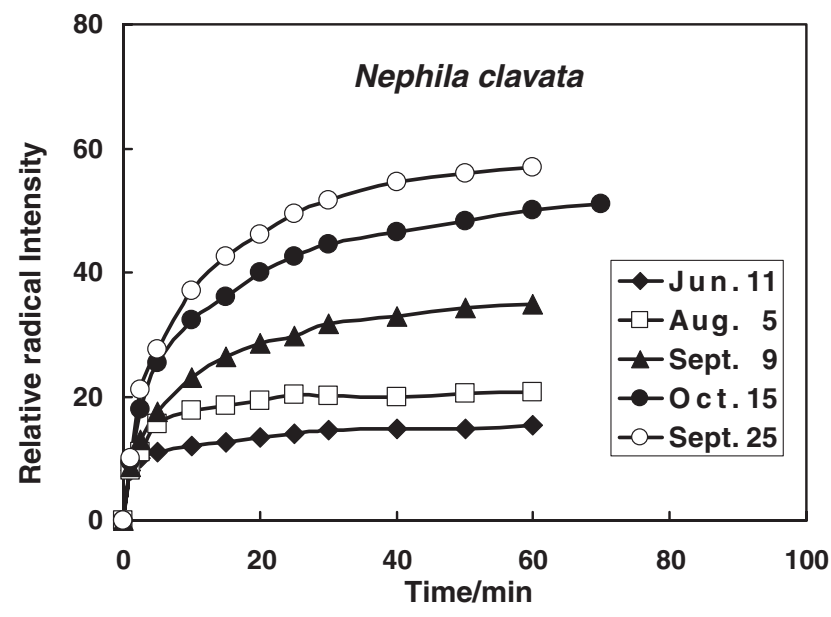

Figure 3. Relative radical intensity of the female $N$. clavata draglines collected at different stages of growth plotted against the duration of UV irradiation. The relative radical intensity is corrected by the sample weight.

Figure 3 shows the relative radical intensity of $N$. clavata draglines collected at different growth stages in Matsue in 1996 plotted against the duration of UV irradiation. Relative radical intensity is defined as the signal intensity corrected by the sample weight of draglines at a given time. The radical intensity showed a rapid initial increase, which gradually slowed as it approached an asymptote with a definite value. The definite value for mature spiders was higher than for juveniles. This indicates that UV irradiation caused chemical decomposition of the protein molecules comprising the dragline, thereby increasing the number of radicals produced over time. In addition, the number of radicals in the draglines of mature spiders was larger than in those of juveniles, which indicates that the draglines of the mature spiders in autumn degraded more easily than those of the juvenile spiders in June. The effect of aging is therefore of im- 


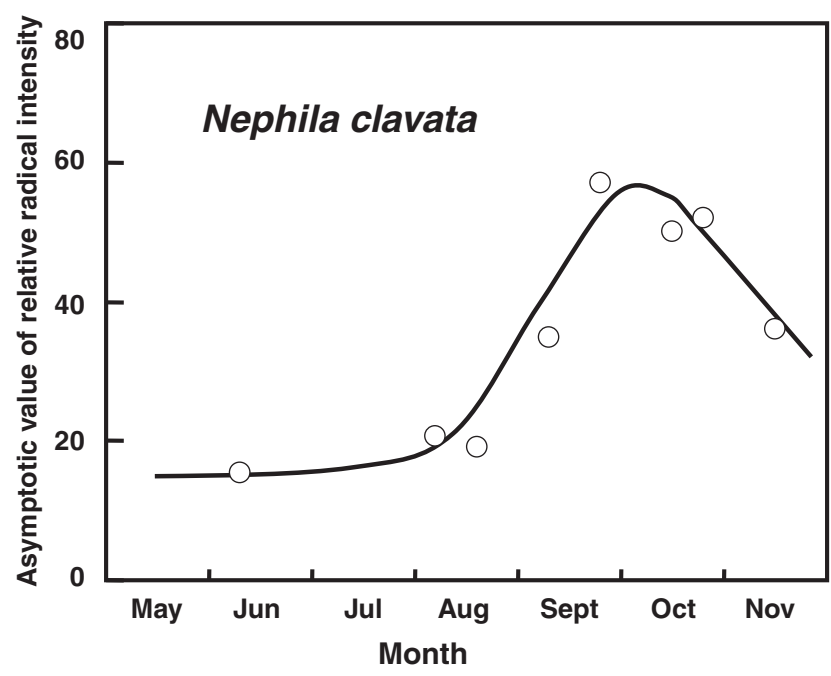

Figure 4. Asymptotic values of relative radical intensity for $N$. clavata draglines collected at different stages of growth and exposed to UV rays. The relative radical intensity is corrected by sample weight. The maximum radical intensity was observed around the end of September and the beginning of October.

portance to the properties of spider silks. The absolute radical concentrations at the definite values were estimated to be in the order of $10^{14}$ spins $/ \mathrm{mg}$, which roughly corresponded to one radical per $\sim 10000 \mathrm{ami}-$ no acids.

Figure 4 shows the asymptotic relative radical intensity values of draglines collected at different stages of growth, which were measured immediately after UV irradiation. The relative radical intensity began to increase in August and peaked around the end of September and the beginning of October, after which it decreased. The maximum relative radical intensity cannot be explained in terms of aging alone; therefore, we compared the relative radical intensity with the optical absorbance of the draglines.

Figure 5 shows the optical reflectance spectra for wavelengths between 190 and $700 \mathrm{~nm}$ for female $N$. clavata draglines collected in August, September and October. In general, the small value of optical reflectance corresponds to the large value of absorbance even though the effects of transmitted intensity through samples cannot be neglected. Thus, minimums of the optical reflectance at $\sim 450 \mathrm{~nm}, 350 \mathrm{~nm}$ and $275 \mathrm{~nm}$ mean the absorption of waves. The absorbance at $\sim 450 \mathrm{~nm}$ corresponded to the yellowing of draglines in autumn. ${ }^{6}$ The absorbance observed at $\sim 275 \mathrm{~nm}$ became intense around September and October, accompanied by an increase in the absorbance at $\sim 350$ and $450 \mathrm{~nm}$. The wavelength of $\sim 275 \mathrm{~nm}$ is within the range (250-365 nm) of the UV irradiation used in the ESR measurements.

The relationship between optical absorbance and month was similar to that observed for relative radical

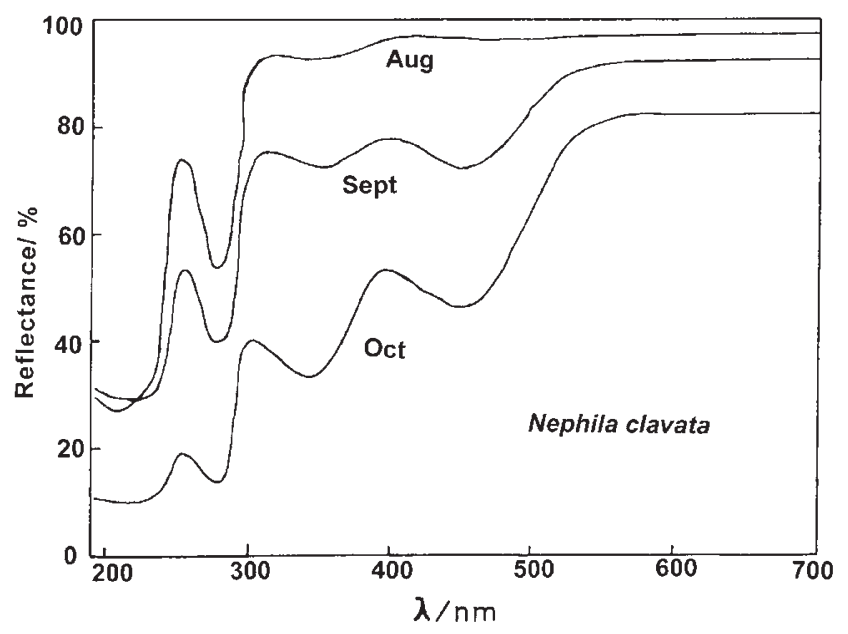

Figure 5. Reflection spectra in the region of 190-700 nm for N. clavata draglines collected in August, September and October. Reflection was low at wavelengths of approximately 275, 350 and $450 \mathrm{~nm}$; the absorbance at $\sim 275 \mathrm{~nm}$ was particularly notable. The absorbance at $\sim 450 \mathrm{~nm}$ corresponds to the yellowing of draglines in autumn.

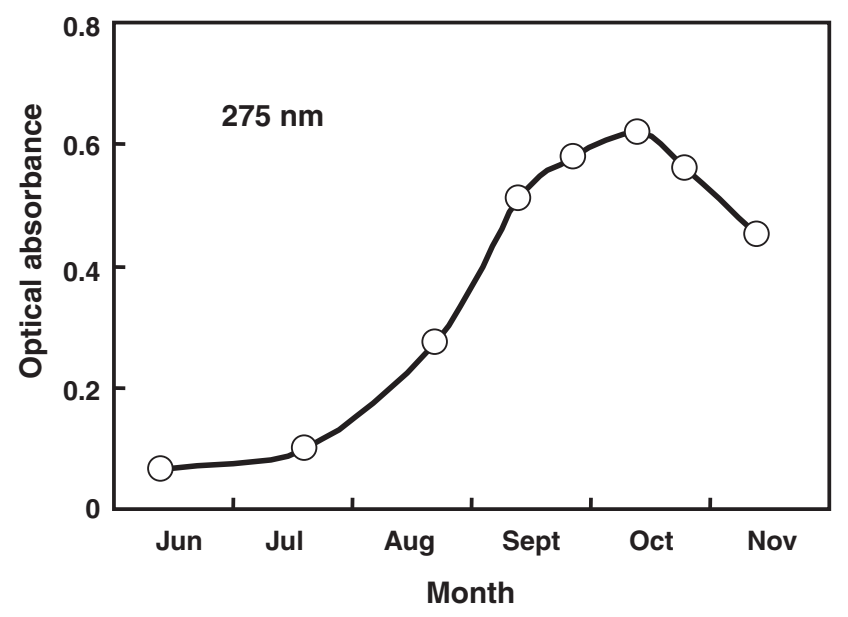

Figure 6. Optical absorbance at $\sim 275 \mathrm{~nm}$ for draglines collected at different stages of growth by month. The maximum optical absorbance was observed in October.

intensity (Figure 4). The optical absorbance at $\sim 275 \mathrm{~nm}$ for draglines collected at different stages of growth reached its largest value in October, as shown in Figure 6. This indicates that the draglines absorbed easily the most UV radiation with a wavelength $\sim 275 \mathrm{~nm}$ around September and October. Generally, exposure to UV radiation induces the degradation of silk. Therefore UV irradiation with a wavelength $\sim 275 \mathrm{~nm}$ might most effectively degrade the protein molecules constituting draglines during this month. The silk from juvenile spiders was more resistant to exposure to UV radiation because it absorbed less UV rays with a wavelength $\sim 275 \mathrm{~nm}$. By contrast, the silk from mature spiders was significantly less resistant to UV irradiation because it absorbed more UV rays at this wavelength. 


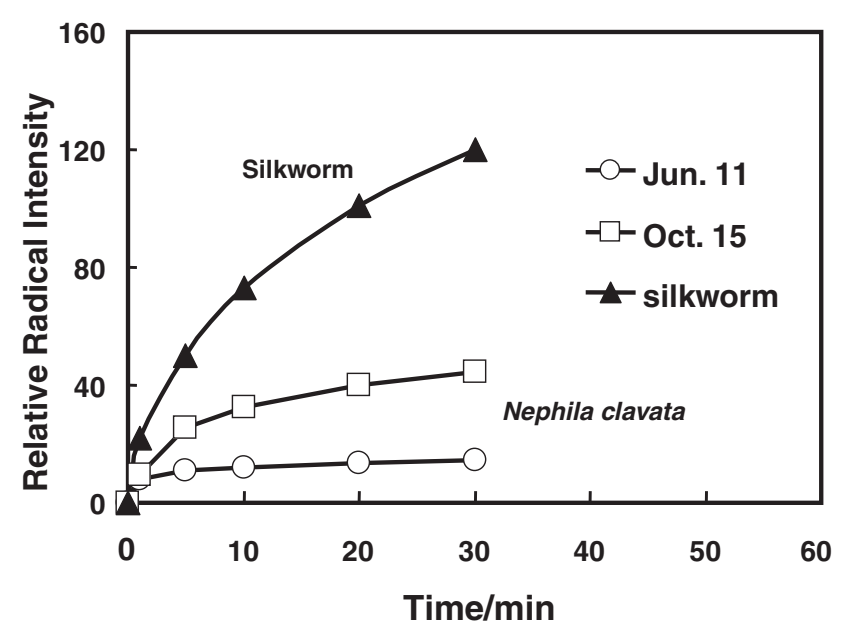

Figure 7. Relationship between relative radical intensity and duration of UV irradiation in draglines and in silks produced by silkworm. The draglines were prepared from juvenile and mature $N$. clavata collected in June 11 and October 15, respectively.

We also compared the resistance to UV irradiation of silk produced by spiders with that produced by silkworm. Figure 7 shows the relationship between radical intensity and the duration of UV irradiation for draglines from juvenile and mature $N$. clavata and for silks prepared from silkworm. The radical intensity of the draglines of juvenile spider was lower than those of mature spider; however, the radical intensity of the draglines of spiders at both stages was significantly lower than that of the silk produced by silkworms. These results show that the draglines of juvenile spiders have a superior resistance to UV irradiation compared with those of mature spiders, and that spider draglines are more resistant to UV irradiation than the silks of silkworm.

\section{DISCUSSION}

UV irradiation induced the production of $\mathrm{C}_{\alpha}$-centered radicals of the protein molecules constituting spider draglines, which was attributed to the breaking of chemical bonds. Greater numbers of radicals were induced by UV irradiation in mature spiders than in juveniles. The low levels of absorbance of UV radiation by the draglines of juveniles of $N$. clavata might be responsible for their stronger resistance to UV irradiation. The optical reflectance spectra therefore indicated that absorbance at $\sim 275 \mathrm{~nm}$ triggers decomposition of the protein molecules in spider silk.

However, the absorbance of spider silks in the UV region was much lower than that of silks produced by silkworm. Therefore, the draglines of $N$. clavata spiders might have a structure that is more resistant to UV irradiation than those of silkworm. This property might be a consequence of the external environment in which spider silk is produced and functions.

Absorbance at $\sim 275 \mathrm{~nm}$ is mainly attributed to aromatic amino acids, such as tyrosine and tryptophan, in the draglines. Although the absorption coefficient of tryptophan at $275 \mathrm{~nm}$ was fourfold larger than that of tyrosine, ${ }^{23}$ the tryptophan content of spider silks was smaller than a half of tyrosine. Therefore, the absorption might have been mainly due to tyrosine residues constituting draglines. ${ }^{4}$ However, the absorbance at $\sim 275 \mathrm{~nm}$ was also associated with the yellowing of draglines and an increased absorbance at $\sim 450 \mathrm{~nm}$, as shown in Figure 5. Therefore, the tyrosine constituting draglines and the chemical substances giving the yellowing of draglines accelerate the absorption at $\sim 275 \mathrm{~nm}$ and may induce the production of radicals attributed to the decomposition of protein molecules.

The spider silks may be a useful next-generation material for textiles from the viewpoint of the resistance to UV irradiation. At present, however, the mechanism underlying this process is not clear. In addition, the chemical structure of spider silk has not yet been determined. Future studies should clarify both the mechanism of the degradation induced by exposure to UV radiation and the chemical structure of spider silk, which was shown here to have a strong resistance to UV irradiation.

Acknowledgment. We are grateful to Dr. Akihito Hashidzume of Osaka University, Toyonaka, Japan for helps of ESR measurements and also to Professor Mikiharu Kamachi of Fukui University of Technology, Fukui, Japan for useful comments on ESR measurements.

\section{REFERENCES}

1. M. W. Denny, J. Exp. Biol., 65, 483 (1976).

2. R. W. Work and N. Morosoff, Text Res. J., 46, 349 (1982).

3. J. M. Gosline, M. W. Denny, and M. E. DeMond, Nature, 309, 551 (1984).

4. S. Osaki, Acta Arachnol., 37, 69 (1989).

5. F. Vollrath and D. Edmonds, Nature, 340, 305 (1989).

6. S. Osaki, Acta Arachnol., 38, 21 (1989).

7. M. A. Becker, D. V. Mahoney, P. G. Lenhert, R. K. Eby, D. Kaplan, and W. W. Adams, "Silk Polymers," ACS Symposium Series 544, Washington, D.C., 1995, p 185.

8. P. M. Cunniff, S. A. Fossey, M. A. Auerbach and J. W. Song, "Silk Polymers," ACS Symposium Series 544, Washington, D. C., 1995, p 234.

9. A. M. Simmons, C. A. Michal, and L. W. Jelinski, Science, 271, 84 (1996).

10. F. Vollrath and D. P. Knight, Nature, 29, 541 (2001).

11. S. Osaki and R. Ishikawa, Polym. J., 34, 25 (2002).

12. S. Osaki, Nature, 348, 419 (1996).

13. S. Osaki, Int. J. Biol. Macromol., 24, 283 (1999). 
14. S. Osaki, Polym. J., 35, 261 (2003).

15. M. Xu and R. V. Lewis, Proc. Natl. Acad. Sci. U.S.A., 87, 7120 (1990).

16. M. B. Hinman and R. V. Lewis, J. Biol. Chem., 267, 19320 (1992).

17. A. Lazaris, S. Arcidiacono, Y. Huang, J. F. Zhou, F. Duguay, N. Chretien, E. A. Welsh, J. W. Soares, and C. N. Karatzas, Science, 295, 472 (2002).

18. J. A. Weil, J. R. Bolton, and J. E. Wertz, "Electron Paramagnetic Resonance," Wiley-Interscience, New York,
N.Y. (1994).

19. A. Kajiwara, A. K. Nanda, and K. Matyjaszewski, Macromolecules, 37, 1378 (2004).

20. S. Osaki, Acta Arachnol., 43, 1 (1994).

21. S. Osaki, Acta Arachnol., 46, 1 (1997).

22. A. Rauk, D. Yu, J. Taylor, G. V. Shustov, D. A. Block, and D. A. Armstrong, Biochemistry, 38, 9089 (1999).

23. G. D. Fasman, "Practical Handbook of Biochemistry and Molecular Biology," CRC Press LLC, Boca Raton, FL, 1989, p 81. 